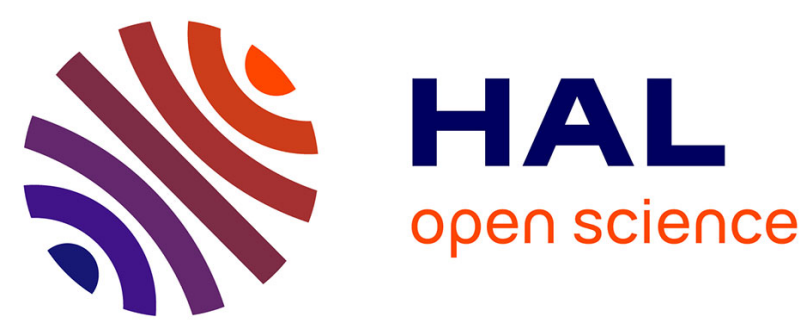

\title{
Nonlinear Characterization of Waveguide Index Profile: Application to Soft-Proton-Exchange in LiNbO_3
}

Maxim Neradovskiy, Herve Tronche, Dmitry Chezganov, Elena A Pashnina, Evgeniy O Vlasov, Pascal Baldi, Tommaso Lunghi, Vladimir Ya Shur, Florent Doutre, Marc P de Micheli

\section{To cite this version:}

Maxim Neradovskiy, Herve Tronche, Dmitry Chezganov, Elena A Pashnina, Evgeniy O Vlasov, et al.. Nonlinear Characterization of Waveguide Index Profile: Application to Soft-ProtonExchange in LiNbO_3. Journal of Lightwave Technology, 2021, 39 (14), pp.4695-4699. 10.1109/JLT.2021.3077637 . hal-03390614

\section{HAL Id: hal-03390614 https://hal.science/hal-03390614}

Submitted on 21 Oct 2021

HAL is a multi-disciplinary open access archive for the deposit and dissemination of scientific research documents, whether they are published or not. The documents may come from teaching and research institutions in France or abroad, or from public or private research centers.
L'archive ouverte pluridisciplinaire HAL, est destinée au dépôt et à la diffusion de documents scientifiques de niveau recherche, publiés ou non, émanant des établissements d'enseignement et de recherche français ou étrangers, des laboratoires publics ou privés. 


\title{
Nonlinear Characterization of Waveguide Index Profile: Application to Soft-Proton-Exchange in $\mathrm{LiNbO}_{3}$
}

\author{
Maxim Neradovskiy, Hervé Tronche, Dmitry S. Chezganov, Elena A. Pashnina, Evgeniy O. Vlasov, Pascal Baldi, \\ Tommaso Lunghi, Vladimir Ya. Shur, Florent Doutre, and Marc P. De Micheli
}

\begin{abstract}
In integrated photonics, the precise knowledge of the waveguides refractive index profile is mandatory for the modeling of photonic chips and therefore implementing innovative circuits. Usual index profile determination relies on effective index measurement of propagating modes in planar waveguides coupled with numerical fitting tools. In this paper we propose an alternative technique based on the characterization of the second harmonic generation signature of a nonlinear waveguide. We include the characterization of high-order spatial modes showing their relevance to probe both vertical and lateral distributions. We finally provide an explicit profile ready-to-use for modeling soft-proton exchanged waveguides in lithium niobate and we test its prediction capability.
\end{abstract}

\section{INTRODUCTION}

A NNEALED-PROTON exchange (APE) [1] and softproton exchange (SPE) [2] in lithium niobate (LN) are well-known fabrication techniques to create shallow optical waveguides [3], [4]. The integration of several components on the same chip requires repeatable fabrication process and precise knowledge of the refractive index profile in order to control and predict the optical performances [5]. All the current techniques attempting to reconstruct the profile of a proton-exchanged waveguide rely on the characterization of the effective refractive indices of the guided modes [1], [5][9]. This measurement is performed at shorter wavelengths $(\lesssim 633 \mathrm{~nm})$ on planar, multi-mode waveguides by means of m-line (dark or bright) spectroscopy [10]. In a subsequent step, an algorithm optimizes the refractive index profile by comparing the experimental results with the expected, calculated ones [11]. The optimization is realized by adjusting the refractive index profile according to a primitive function. Initially simple analytical functions have been proposed (e. g. step [12], gaussian [13], exponential [14]) but their prediction capabilities were limited since these functions do not capture the complex dynamics of the diffusion process occurring during both APE and SPE. A more sophisticated generalized Gaussian function has been later introduced [15] resulting in more accurate results. Today, the most precise model simulates

M. Neradovoskiy, H. Tronche, D. Chezganov, P. Baldi, T. Lunghi, F. Doutre and M. De Micheli are with Université Côte d'Azur, CNRS, Institut de Physique de Nice, Parc Valrose, 06108 Nice Cedex 2, France e-mail: (florent.doutre@inphyni.cnrs.fr).

M. Neradovoskiy, D. Chezganov, E. Pashnina, E. Vlasov and V. Shur are with School of Natural Sciences and Mathematics, Ural Federal University, 620000 Ekaterinburg, Russia.

Manuscript received January 27, 2021. the kinetics of protons in the substrate by solving a onedimensional nonlinear diffusion equation [1], [5]. Recently this model has been extended including the anisotropic diffusion properties of proton exchange [9]. Although this method is extremely precise, it is also time-consuming and complex to implement and to reproduce.

Integrated nonlinear converters are the most common devices realized with periodically poled LN (PPLN) waveguides, that relies on the engineering of the ferroelectric domains in the LN crystal [16]. Among the permitted nonlinear conversion processes, Second-Harmonic Generation (SHG) corresponds to the conversion of two pump photons with identical frequency toward one harmonic photon with twice the frequency of the initial photons. This process needs to satisfy both energy and momentum conservation which in PPLN waveguides are satisfied through quasi-phase-matching (QPM). Usually, channel waveguides support several spatial modes in the near IR (below $1.0 \mu \mathrm{m}$ ), each of them having quiet different effective indices, therefore, multiple QPM are possible at the same time for different pump wavelengths, generating their harmonic in the $\mathrm{n}^{\text {th }}$ order mode [17], [18]. As we show in this paper, the complete mapping of the SHG signature can provide a detailed feedback about the possible different QPM, allowing to reconstruct the refractive index profile.

In this letter, we introduce a technique relying on SHG spectroscopy that precisely allows to characterize the refractive index profile of a channel waveguide. This profile is found by matching the measured SHG signal with the one allowed by the QPM conditions. The theoretical profile to be fitted is a combination of a generalized Gaussian function in depth and an hyper-Gaussian in width. In addition to previous work [17], [18], we use this method to successfully predict the experimental nonlinear conversion pump-wavelengths / mode-orders for different poling periods on different samples. Although our approach and the current work has been specifically designed for SPE on LN, it can be extended to any other fabrication techniques and nonlinear materials.

\section{SAMPLE FABRICATION}

In the following, we will consider only PPLN waveguides fabricated with SPE technique on congruent LN substrates. The fabrication starts with the deposition of a hard mask of $\mathrm{SiO}_{2}$ on top of the $-\mathrm{Z}$ face of the substrate. Through a liftoff, several openings are created on the silica mask. 
These openings are then submitted to proton/lithium ionic substitution. For simplification, the waveguide width labelling is the span of these openings, without considering the proton lateral diffusion. The proton exchange is performed in a sealed zirconium container filled with a powder mixture of benzoic acid buffered with lithium benzoate for 72 hours at $300^{\circ} \mathrm{C}$. The waveguides investigated in this work are multi-mode in the short IR regions $(700-825 \mathrm{~nm})$ and single-mode between $1400 \mathrm{~nm}$ and $1650 \mathrm{~nm}$. SPE waveguides guide only transverse magnetic (TM) modes that will be labeled using the usual convention for rectangular waveguides: $\mathrm{TM}_{x y}$ with $\mathrm{x}(\mathrm{y})$ the mode order in lateral (vertical) direction.

Once the waveguides fabricated, the silica mask is removed, and we create the periodical poling by electron beam (ebeam) writing technique [19], [20] which guarantees a high resolution control over the poling period at a price of a limited poling area. The scanning electron microscope (Carl Zeiss Auriga CrossBeam workstation) with Schottky field emission gun equipped with the e-beam lithography (EBL) system (Raith Elphy Multibeam) was used for e-beam domain patterning. The exposure parameters and e-beam positioning were controlled by EBL system. The irradiation pattern was specified by the Raith Nanosuite software. Before irradiation, a $2.5 \mu \mathrm{m}$-thick layer of negative e-beam resist (Micro Chemicals AZnLOF 2020) was deposited by spincoating on the surface to be irradiated and a copper electrode was deposited by magnetron sputtering on the opposite polar surface. The irradiation was performed at accelerating voltage of $10 \mathrm{kV}$ and e-beam current of $1.5 \mathrm{nA}$. The patterns with length of $1.5 \mu \mathrm{m}$ consisting of rectangular stripes with various periods were used. SHG spectra were characterized for waveguides width of $5,6,7$ and $8 \mu \mathrm{m}$.

\section{NONLINEAR CHARACTERIZATION}

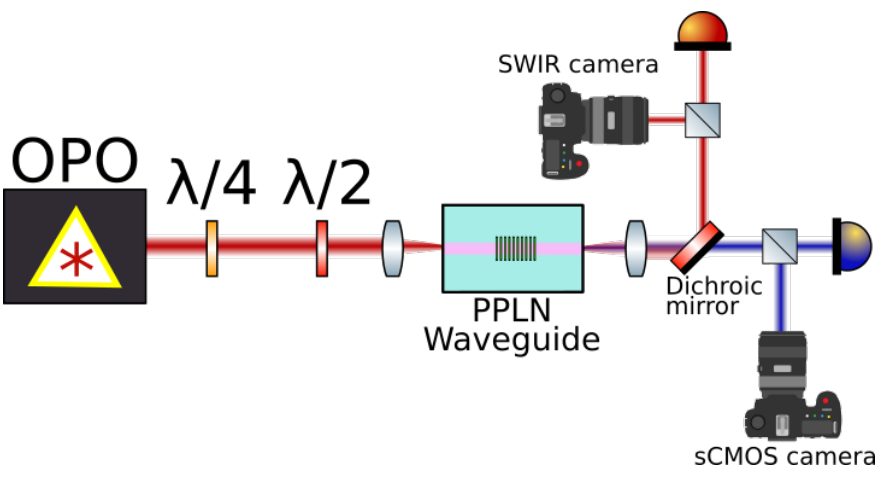

Fig. 1. Schematics of the SHG characterization setup. OPO laser beam is controlled in polarisation and then focused in the waveguide to be tested. At sample output, the pump is separted from its harmonic using a dichroic mirror. On each arm, detection is made using a camera and an energymeter.

The experimental setup is represented in Fig. 1. The sample is a $15 \mathrm{~mm}$-long waveguide with a poling region of $1.5 \mathrm{~mm}$ length and a poling period of $15.8 \mu \mathrm{m}$. The sample was injected with an optical beam generated by a pulsed OPO (Ekspla NT242) with $4 \mathrm{~ns}$ pulses at $1 \mathrm{kHz}$ repetition rate and a wavelength tunablility between 220 and $2600 \mathrm{~nm}$ ). At the output, the collected beams at $\lambda_{\omega}$ and $\lambda_{2 \omega}$ were spectrally separated and sent either on an energymeter (Coherent LabMax TOP) to measure pulses' energy or a camera (Photonic Science sCMOS and SWIR) to visualize the spatial distribution of the mode. Fig. 2 reports the SH spectra for the different waveguide widths. We determine $\lambda_{\omega}$, the QPM pump central wavelength, using a fitting of the experimental data by a $\operatorname{sinc}^{2}(\lambda)$ function, as shown in the inset of Fig. 2. Given the high precision when fitting of the sideband zeros and maxima, the estimation precision is $\Delta \lambda_{\omega}= \pm 0.2 \mathrm{~nm}$.

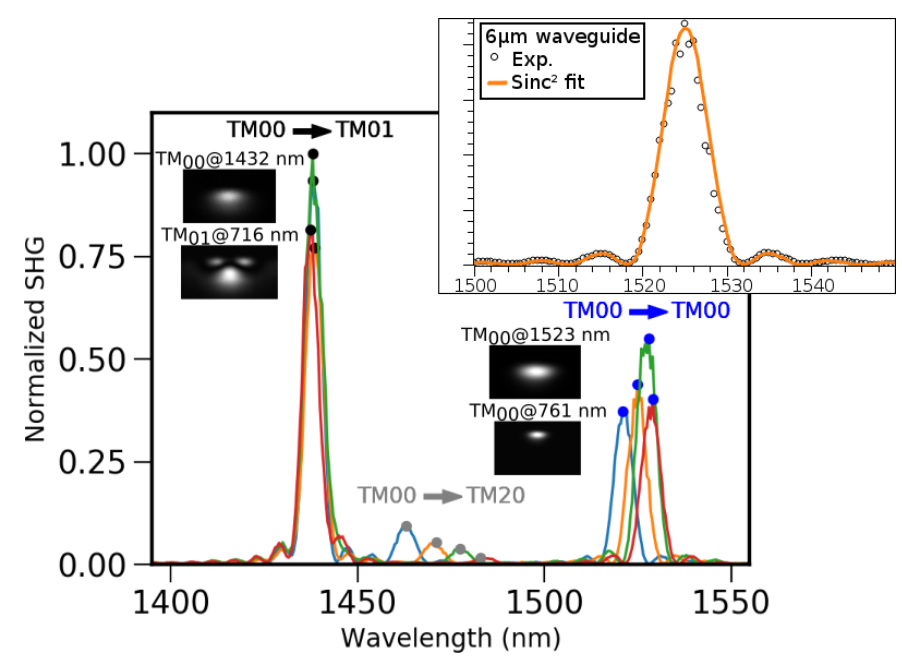

Fig. 2. SHG intensity in function of the pump wavelength for different waveguide width. Each peak for a waveguide correspond to a phase-matching with different order of the harmonic mode. Inset: example of SHG central wavelength determination for the $1525 \mathrm{~nm}$ peak of $6.0 \mu \mathrm{m}$ waveguide, dots: experimental data, color line: $\operatorname{sinc}^{2}(\lambda)$ fitting function.

For each waveguide, we distinguish three phase-matched signatures, which correspond to the conversion of the fundamental mode $\mathrm{TM}_{00}^{\omega}$ to 3 different spatial modes: $\mathrm{TM}_{00}^{2 \omega}$, $\mathrm{TM}_{20}^{2 \omega}$, and $\mathrm{TM}_{01}^{2 \omega}$ (from right to the left in Fig.2). We verified that $\mathrm{TM}_{01}$ mode acquired in the visible range by CMOS camera is not a composition of several modes by comparing it to a numerically calulculated mode (Fig. 3) using Lumerical MODE software.

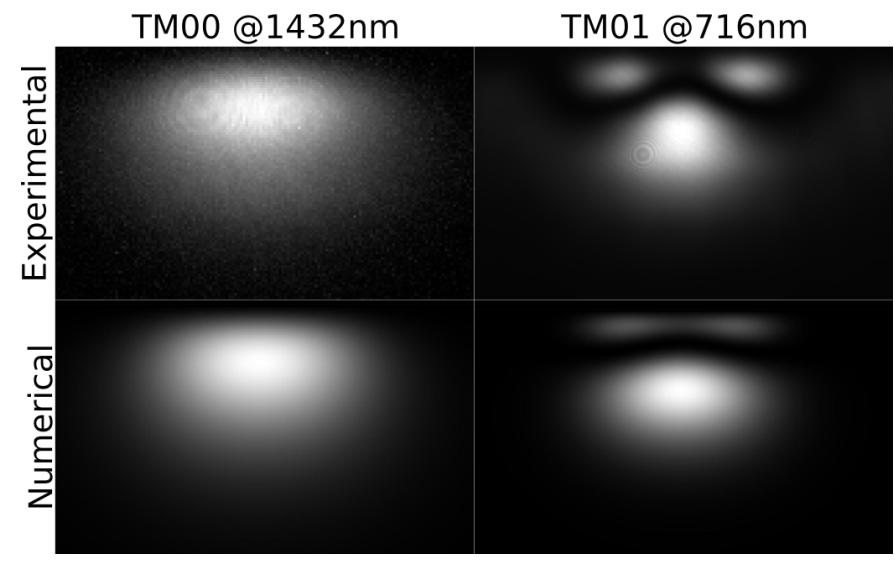

Fig. 3. Modes comparison for fundamental (left) and harmonic (right) between CMOS acquisition (top) and numerical simulation (bottom). 
TABLE I

PUMP WAVELENGTHS ALLOWING QPM FOR DIFFERENT HARMONIC TRANSVERSE MODES AND WAVEGUIDE WIDTHS. EXPERIMENTAL RESULTS (EXP.) ARE COMPARED TO THE ONE OBTAINED USING NUMERICAL PROFILES $\left(n_{1}, n_{2}, n_{2}^{*}\right)$ THROUGH THE ABSOLUTE DIFFERENCE BETWEEN CALCULATED AND MEASURED WAVELENGTHS $\left(\Delta_{1}, \Delta_{2}, \Delta_{2}^{*}\right)$. $n_{2}^{*}$ IS A PARTICULAR CASE FOR WHICH ONLY THE TM $\mathrm{TM}_{00}^{\omega} \Rightarrow \mathrm{TM}_{00}^{2 \omega}$ CONVERSION IS OPTIMIZED.

\begin{tabular}{|c|c|c|c|c|c|c|c|c|}
\hline \multirow{2}{*}{$\begin{array}{l}\text { SHG } \\
\text { spatial } \\
\text { mode }\end{array}$} & \multirow{2}{*}{$\begin{array}{c}\mathrm{w}_{\text {mask }} \\
(\mu \mathrm{m})\end{array}$} & \multirow{2}{*}{$\begin{array}{c}\text { Exp. } \\
\lambda_{\omega}( \pm 0.2 n m)\end{array}$} & \multicolumn{2}{|c|}{$\mathrm{n}_{2}^{*}(\mathrm{x}, \mathrm{y}, \lambda)$} & \multicolumn{2}{|c|}{$n_{1}(x, y, \lambda)$} & \multicolumn{2}{|c|}{$n_{2}(x, y, \lambda)$} \\
\hline & & & $\lambda_{\omega, 2}^{*}(\mathrm{~nm})$ & $\Delta_{2}^{*}(\mathrm{~nm})$ & $\lambda_{\omega, 1}(\mathrm{~nm})$ & $\Delta_{1}(\mathrm{~nm})$ & $\lambda_{\omega, 2}(\mathrm{~nm})$ & $\Delta_{2}(\mathrm{~nm})$ \\
\hline \multirow{4}{*}{$\mathrm{TM}_{00}$} & 8 & 1529.0 & 1528.9 & 0.1 & 1526.1 & 2.9 & 1529.2 & 0.2 \\
\hline & 7 & 1528.0 & 1527.9 & 0.1 & 1526.4 & 1.6 & 1527.9 & 0.1 \\
\hline & 6 & 1525.0 & 1525.8 & 0.8 & 1527.7 & 2.7 & 1526.0 & 1.0 \\
\hline & 5 & 1521.0 & 1522.0 & 1.0 & 1526.9 & 5.9 & 1523.0 & 2.0 \\
\hline \multirow{4}{*}{$\mathrm{TM}_{20}$} & 8 & 1483.0 & 1468.5 & 14.5 & 1482.0 & 1.0 & 1483.9 & 0.9 \\
\hline & 7 & 1477.5 & 1460.0 & 17.5 & 1477.2 & 0.3 & 1476.6 & 0.9 \\
\hline & 6 & 1471.0 & 1452.5 & 18.5 & 1471.1 & 0.1 & 1468.2 & 2.8 \\
\hline & 5 & 1463.0 & 1451.4 & 11.6 & 1463.7 & 0.7 & 1458.8 & 4.2 \\
\hline \multirow{4}{*}{$\mathrm{TM}_{01}$} & 8 & 1437.5 & 1440.7 & 3.2 & 1439.6 & 2.1 & 1440.5 & 3.0 \\
\hline & 7 & 1438.0 & 1431.7 & 6.3 & 1439.7 & 1.7 & 1439.7 & 1.7 \\
\hline & 6 & 1438.5 & 1425.6 & 12.9 & 1440.5 & 2.0 & 1440.3 & 1.8 \\
\hline & 5 & 1438.0 & 1422.1 & 15.9 & 1434.8 & 3.2 & 1434.5 & 3.5 \\
\hline \multicolumn{3}{|c|}{$\overline{\sum \Delta}$} & & $\overline{102.4}$ & & 24.3 & & 22.1 \\
\hline
\end{tabular}

The different transverse modes, waveguide widths, and $\lambda_{\omega}$ are presented in the three first columns of Table I. Let us notice that the wavelengths at which the mode $\mathrm{TM}_{01}^{2 \omega}$ is generated are almost identical, whatever the waveguide width. This is connected with the condition of non-critical quasi-phasematching [21] and will be discussed further in another article.

\section{INDEX PROFILE DETERMINATION}

We assume the very general shape of the index profile in the waveguide to be a function of the transverse position $(x, y)$ and wavelength $(\lambda)$ :

$$
\begin{aligned}
n(x, y, \lambda) & =n_{\text {sell }}(\lambda)+\Delta n(\lambda, x, y) \\
& =n_{\text {sell }}(\lambda)+\delta n(\lambda) \cdot f(y) \cdot g(x)
\end{aligned}
$$

where $n_{\text {sell }}$ is the refractive extraordinary index of the unexchanged substrate given by the Sellmeier equations [22] and $\Delta n(\lambda, x, y)$ is the increase of the refractive index induced by proton-exchange. It can be decomposed in a product of the spatial distribution functions along the vertical $(f(y))$ and lateral $(g(x))$ directions and a spectral distribution function $\delta n(\lambda)$. The spectral distribution is modeled using a Sellmeierlike function [23].

$$
\delta n(\lambda)=\delta n_{0}+\frac{\delta n_{1}}{\lambda^{2}}+\frac{\delta n_{2}}{\lambda^{4}} .
$$

The depth distribution is a generalized Gaussian function, as proposed in [15]:

$$
f(y)=\exp \left[-\left(\frac{y}{w_{d}}\right)^{\gamma}\right],
$$

in which $w_{d}$ and $\gamma$ are the waveguide depth and the profile's slope, respectively. We compare two different distributions describing the lateral proton diffusion:

$$
\begin{aligned}
& g_{1}(x)=\exp \left[-\left(\frac{x}{w_{l}+w_{\text {mask }} / 2}\right)^{\alpha}\right] \\
& g_{2}(x)=\left[1+\exp \left(\frac{|x|-w_{\text {mask }} / 2-L}{w_{l}}\right)\right]^{-1}
\end{aligned}
$$

$w_{\text {mask }}$ is the opening width in the silica hard mask. $w_{l}$ is a parameter related to the lateral diffusion of proton below the silica mask. $g_{1}(x)$ is also a generalized Gaussian function, with a slope $\alpha . g_{2}(x)$ is an empirical formula that we introduced within this work, which contains a free parameter, $L$. The differences between these two equations will be detailed in the following. From now, we will call $n_{i}(x, y, \lambda)$ the refractive index distribution calculated using the lateral distribution $g_{i}(x)$.

Using Eq.(2), we can calculate the effective refractive index of the propagating modes and compare them to the experimental ones through the satisfaction of energy conservation rule and quasi-phase matching condition, i.e.:

$$
\lambda_{\omega}=2 \Lambda\left[n_{i j}\left(\lambda_{2 \omega}\right)-n_{00}\left(\lambda_{\omega}\right)\right]
$$

where $\lambda_{\omega}, \lambda_{2 \omega}$ and $\Lambda$ are the pump wavelength, the harmonic wavelength, and the poling period, respectively. The indices $i, j$ label the harmonic mode orders. We then use an homemade algorithm that varies once at a time each of the seven free parameters of our model, namely $\delta n_{0}, \delta n_{1}, \delta n_{2}, w_{d}$, $\gamma, w_{l}, \alpha$ (or $L$ ). Once a maximal fitting is found, an other parameter is varied, and so on. Once all the parameters are optimized, the algorithm loops as long as a significant increase in the fitting between calculated and measured values is possible and therefore get closer to the optimal profile. Given the extensive set of data provided by the different spatial modes, the large number of free parameters is not a limitation. Moreover, the dataset can be extended, if needed, by including other sets of pump/harmonic-wavelengths and mode-orders through different poling periods. The theoretical effective indices have been calculated using Python [24] with the EMpy package [25]. Both experimental and numerical results are reported in Table I.

In a first approach, we limited the algorithm to consider only the $\mathrm{TM}_{00}^{\omega} \Rightarrow \mathrm{TM}_{00}^{2 \omega}$ conversions, as already done in previous published work [5]. The optimal profile, $n_{2}^{*}(x, y, \lambda)$, while correctly matching the fundamental modes interaction $\left(\Delta_{2}^{*} \leq 1 \mathrm{~nm}\right)$, shows a poor fitting for modes $\mathrm{TM}_{20}$ and $\mathrm{TM}_{01}$ 
and does not predict the insensitivity to waveguide's width of modes $\mathrm{TM}_{01}$ experimentally observed in Fig. 2 .

TABLE II

OPTIMIZED NUMERICAL PARAMETERS OF THE INDEX PROFILE FUNCTIONS

\begin{tabular}{|c|c|c|c|}
\hline & $n_{1}(x, y, \lambda)$ & $n_{2}(x, y, \lambda)$ & $n_{2}^{*}(x, y, \lambda)$ \\
\hline \hline$\delta n_{0}$ & 0.0455 & 0.0475 & 0.035 \\
\hline$\delta n_{1}$ & $-7.0 \times 10^{-16}$ & $-6.0 \times 10^{-16}$ & $-4.75 \times 10^{-16}$ \\
\hline$\delta n_{2}$ & $1.25 \times 10^{-27}$ & $1.2 \times 10^{-27}$ & $1.29 \times 10^{-27}$ \\
\hline$w_{l}$ & $1.35 \times 10^{-6}$ & $0.975 \times 10^{-6}$ & $0.975 \times 10^{-6}$ \\
\hline$L$ & - & $0.95 \times 10^{-6}$ & $0.1 \times 10^{-6}$ \\
\hline$\alpha$ & 4.0 & - & - \\
\hline$w_{d}$ & $0.5 \times 10^{-6}$ & $0.49 \times 10^{-6}$ & $1.175 \times 10^{-6}$ \\
\hline$\gamma$ & 0.55 & 0.55 & 0.55 \\
\hline
\end{tabular}

However, when the entire SHG signature is taken into account by the algorithm, both $n_{1}(x, y, \lambda)$ and $n_{2}(x, y, \lambda)$ match correctly the experimental results (the optimal parameters value for $n_{1}, n_{2}$ and $n_{2}^{*}$ are presented in Table II). More specifically, $n_{2}(x, y, \lambda)$ results in more accurate estimations, fitting better the nonlinear processes for the mode $\mathrm{TM}_{00}$, the refractive index profile is shown in Fig. 4. This higher precision is linked to the fact that $g_{1}(x)$ is a normalized function, while the $g_{2}(0)$ value depends on the waveguide width. We stress that this lower mismatch is more than a numerical artifact, as experimentally verified before [26], [27].

In order to verify the inference capability of our model, the

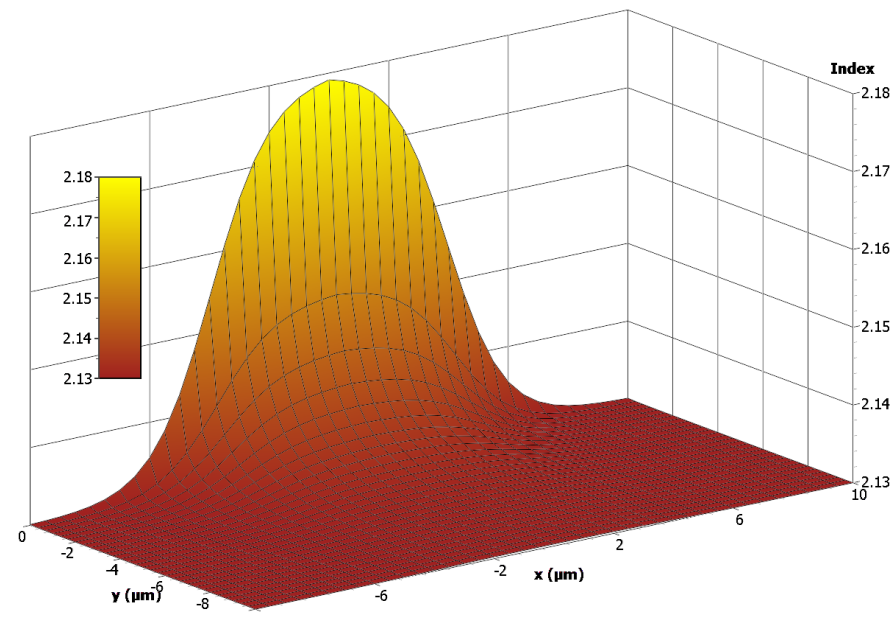

Fig. 4. Refractive index profile $n_{2}(x, y, \lambda)$

poling period meeting the QPM condition for SHG in the $\mathrm{TM}_{01}$ mode with a $\mathrm{TM}_{00}$ pump in the telecom spectral $\mathrm{C}$ band is calculated to be $18.0,19.0$ and $19.5 \mu \mathrm{m}$. We fabricated a second batch of samples with similar proton exchange parameters using e-beam writing. Additionally, poling period of $16.0 \mu \mathrm{m}$, corresponding to SHG in the mode $\mathrm{TM}_{00}^{\omega}$ with a pump@ $1550 \mathrm{~nm}$ is added as a fabrication quality check. We characterized $\lambda_{\omega}$ for the different spatial modes. Table III reports the error $\Delta_{2}$ for different waveguide widths, poling periods and spatial modes. For $\lambda_{\omega}<1576 \mathrm{~nm}$, the model predicts the SHG wavelength with precision better than $4 \mathrm{~nm}$
TABLE III

PUMP WAVELENGTHS (EXPERIMENTAL AND CALCULATED) ALLOWING QPM FOR DIFFERENT SHG TRANSVERSE MODES, POLING PERIODS AND WAVEGUIDE WIDTHS.

\begin{tabular}{|c|c|c|c|c|c|}
\hline \multirow{3}{*}{$\begin{array}{c}\text { Second } \\
\text { Harmonic } \\
\text { mode }\end{array}$} & \multirow{2}{*}{ Poling } & \multirow{2}{*}{$w_{\text {mask }}$} & \multicolumn{2}{|c|}{$\lambda_{\omega}$} & \multirow{2}{*}{$\Delta_{2}$} \\
\hline & & & exp. & calc. & \\
\hline & \multicolumn{2}{|c|}{$(\mu \mathrm{m})$} & \multicolumn{3}{|c|}{$(\mathrm{nm})$} \\
\hline \multirow{6}{*}{$\mathrm{TM}_{00}$} & \multirow{3}{*}{16.0} & 8 & 1534.9 & 1537.5 & 2.6 \\
\hline & & 7 & 1533.6 & 1536.4 & 2.8 \\
\hline & & 6 & 1531.0 & 1534.4 & 3.4 \\
\hline & \multirow{3}{*}{18.0} & 8 & 1619.1 & 1628.5 & 9.4 \\
\hline & & 7 & 1617.8 & 1626.8 & 9.1 \\
\hline & & 6 & 1615.1 & 1623.5 & 8.4 \\
\hline \multirow{7}{*}{$\mathrm{TM}_{01}$} & \multirow{3}{*}{18.0} & 8 & 1516.9 & 1518.6 & 1.7 \\
\hline & & 7 & 1517.4 & 1519.5 & 2.1 \\
\hline & & 6 & 1517.8 & 1517.5 & 0.3 \\
\hline & \multirow{3}{*}{19.0} & 8 & 1555.8 & 1556.3 & 0.5 \\
\hline & & 7 & 1555.2 & 1556.5 & 1.3 \\
\hline & & 6 & 1555.1 & 1551.4 & 3.7 \\
\hline & 19.5 & 7 & 1572.7 & 1575.6 & 3.0 \\
\hline
\end{tabular}

for any spatial mode. At longer wavelengths $\left(\lambda_{\omega} \approx 1620 \mathrm{~nm}\right)$ we observe a significant discrepancy $(\approx 10 \mathrm{~nm})$. We attribute this imprecision to the poor knowledge of $\delta n(\lambda)$ at these wavelengths. Nevertheless, the general agreement between theoretical and experimental $\mathrm{SH}$ wavelengths reported in this letter opens to precisely engineer complex integrated photonic structures, e. g. adiabatic tapers [28], splitters [29] or nonlinear emissiters [17], [18].

\section{CONCLusion}

We propose a new characterization technique to precisely assess the refractive index profile of a SPE waveguide, easily adaptable to any waveguide fabrication technique based on ion exchange. Our method, alternatively to the $\mathrm{m}$-line spectroscopy, is based on nonlinear interactions. The fitting algorithm adjusts the refractive index profile of the nonlinear waveguide, trying to match its SHG signature. We have shown that higher precision is obtained by considering SHG toward higher-order spatial modes. We provided a primitive function able to correctly fit the experimental results. The optimal distribution predicts correctly $\lambda_{\omega}$ at different poling periods with precision better than $4 \mathrm{~nm}$ and provides quantitative insights about some characteristics of the nonlinear waveguides as well as the physics underlying the fabrication process. Finally, we provide a ready-to-use refractive index profile that can be employed to simulate linear and nonlinear components based on SPE waveguides.

January 26, 2021

\section{ACKNOWLEDGMENT}

The equipment of the Ural Center for Shared Use "Modern nanotechnology" UrFU was used.

The work of e-beam writing of periodic domains on soft proton exchanged waveguides was supported by the Russian Science Foundation (grant № 19-72-00091) and performed 
by D.S. Chezganov, E.A. Pashnina and E.O Vlasov in Ural Federal University.

The waveguides refractive index characterization was performed at Université Côte d'Azur with the participation of D.S. Chezganov and supported by the French government in the frame of the Metchnikov program.

\section{REFERENCES}

[1] M. L. Bortz and M. M. Fejer, "Annealed proton-exchanged linbo3 waveguides," Opt. Lett., vol. 16, no. 23, pp. 1844-1846, Dec 1991. [Online]. Available: http://ol.osa.org/abstract.cfm?URI=ol-16-23-1844

[2] L. Chanvillard, P. Aschiéri, P. Baldi, D. B. Ostrowsky, M. de Micheli, L. Huang, and D. J. Bamford, "Soft proton exchange on periodically poled linbo3: A simple waveguide fabrication process for highly efficient nonlinear interactions," Applied Physics Letters, vol. 76, no. 9, pp. 1089-1091, 2000. [Online]. Available: https://doi.org/10.1063/1.125948

[3] O. Alibart, V. D'Auria, M. D. Micheli, F. Doutre, F. Kaiser, L. Labonté, T. Lunghi, É. Picholle, and S. Tanzilli, "Quantum photonics at telecom wavelengths based on lithium niobate waveguides," Journal of Optics, vol. 18, no. 10, p. 104001, sep 2016. [Online]. Available: https://doi.org/10.1088/2040-8978/18/10/104001

[4] E. L. Wooten, K. M. Kissa, A. Yi-Yan, E. J. Murphy, D. A. Lafaw, P. F. Hallemeier, D. Maack, D. V. Attanasio, D. J. Fritz, G. J. McBrien, and D. E. Bossi, "A review of lithium niobate modulators for fiber-optic communications systems," IEEE Journal of Selected Topics in Quantum Electronics, vol. 6, no. 1, pp. 69-82, 2000.

[5] R. V. Roussev, "Optical-frequency mixers in periodically poled Lithium Niobate materials modeling, materials, and characterization," $\mathrm{Ph} . \mathrm{D}$. dissertation, Stanford University, 2006.

[6] A. P. Rambu, A. M. Apetrei, F. Doutre, H. Tronche, V. Tiron, M. de Micheli, and S. Tascu, "Lithium niobate waveguides with high-index contrast and preserved nonlinearity fabricated by a high vacuum vapor-phase proton exchange," Photon. Res., vol. 8, no. 1, pp. 8-16, Jan 2020. [Online]. Available: http://www.osapublishing.org/prj/ abstract.cfm?URI=prj-8-1-8

[7] T. Lunghi, F. Doutre, G. Legoff, G. Ayenew, H. Tronche, S. Tanzilli, P. Baldi, and M. De Micheli, "Soft-proton exchange on magnesiumoxide-doped substrates: A route toward efficient and power-resistant nonlinear converters," Journal of Applied Physics, vol. 122, no. 1, p. 013103, 2017. [Online]. Available: https://doi.org/10.1063/1.4991474

[8] B. Vincent, R. Kremer, A. Boudrioua, P. Moretti, Y.-C. Zhang, C.-C. Hsu, and L.-H. Peng, "Green light generation in a periodically poled Zn-doped LiNbO3 planar waveguide fabricated by He+ implantation," Appl. Phys. B, vol. 89, no. 2-3, pp. 235-239, nov 2007. [Online]. Available: http://link.springer.com/10.1007/s00340-007-2788-2

[9] F. Lenzini, S. Kasture, B. Haylock, and M. Lobino, "Anisotropic model for the fabrication of annealed and reverse proton exchanged waveguides in congruent lithium niobate," Opt. Express, vol. 23, no. 2, pp. 1748-1756, Jan 2015. [Online]. Available: http://www. opticsexpress.org/abstract.cfm?URI=oe-23-2-1748

[10] R. Ulrich and R. Torge, "Measurement of Thin Film Parameters with a Prism Coupler," Appl. Opt., vol. 12, no. 12, p. 2901, dec 1973. [Online]. Available: https://www.osapublishing.org/abstract.cfm?URI= ao-12-12-2901

[11] Y. N. Korkishko, V. A. Fedorov, E. A. Baranov, M. V. Proyaeva, T. V. Morozova, F. Caccavale, F. Segato, C. Sada, and S. M. Kostritskii, "Characterization of $\alpha$-phase soft proton-exchanged LiNbO_3 optical waveguides," J. Opt. Soc. Am. A, vol. 18, no. 5, p. 1186, may 2001. [Online]. Available: https://www.osapublishing.org/abstract.cfm? URI=josaa-18-5-1186

[12] K.-K. Wong, "Integrated Optical Waveguides And Devices Fabricated By Proton Exchange: A Review," in Integr. Opt. Circuit Eng. VI, M. A. Mentzer, Ed., vol. 0993. SPIE, dec 1988, p. 13. [Online]. Available: http://proceedings.spiedigitallibrary.org/proceeding. aspx?doi=10.1117/12.960065

[13] N. Goto and G. L. Yip, "Characterization of proton-exchange and annealed $\mathrm{LiNbO}_{3}$ waveguides with pyrophosphoric acid," Appl. Opt., vol. 28, no. 1, p. 60, jan 1989. [Online]. Available: https://www.osapublishing.org/abstract.cfm?URI=ao-28-1-60
[14] M. De Micheli, M. Papuchon, J. Botineau, S. Neveu, P. Sibillot, and D. B. Ostrowsky, "Independent control of index and profiles in proton-exchanged lithium niobate guides," Opt. Lett., vol. 8, no. 2, p. 114, feb 1983. [Online]. Available: https://www.osapublishing.org/ abstract.cfm?URI=ol-8-2-114

[15] J. Nikolopoulos and G. L. Yip, "Accurate modeling of the index profile in annealed proton-exchanged LiNbO 3 waveguides," in Integr Opt. Circuits, K. K. Wong, Ed., vol. 1583, dec 1991, p. 71. [Online]. Available: http://proceedings.spiedigitallibrary.org/proceeding. aspx?doi $=10.1117 / 12.50877$

[16] G. D. Miller, R. G. Batchko, W. M. Tulloch, D. R. Weise, M. M. Fejer, and R. L. Byer, " $42 \%$-efficient single-pass cw secondharmonic generation in periodically poled lithium niobate," Opt. Lett., vol. 22, no. 24, pp. 1834-1836, Dec 1997. [Online]. Available: http://ol.osa.org/abstract.cfm?URI=ol-22-24-1834

[17] A. Christ, K. Laiho, A. Eckstein, T. Lauckner, P. J. Mosley, and C. Silberhorn, "Spatial modes in waveguided parametric downconversion," Phys. Rev. A, vol. 80, p. 033829, Sep 2009. [Online]. Available: https://link.aps.org/doi/10.1103/PhysRevA.80.033829

[18] M. Karpiński, C. Radzewicz, and K. Banaszek, "Experimental characterization of three-wave mixing in a multimode nonlinear KTiOPO4 waveguide," Appl. Phys. Lett., vol. 94, no. 18, pp. 1-4, 2009.

[19] D. S. Chezganov, E. O. Vlasov, M. M. Neradovskiy, L. V. Gimadeeva, E. A. Neradovskaya, M. A. Chuvakova, H. Tronche, F. Doutre, P. Baldi, M. P. De Micheli, and V. Y. Shur, "Periodic domain patterning by electron beam of proton exchanged waveguides in lithium niobate," Applied Physics Letters, vol. 108, no. 19, p. 192903, 2016. [Online]. Available: https://doi.org/10.1063/1.4949360

[20] V. Y. Shur, D. S. Chezganov, A. R. Akhmatkhanov, and D. K. Kuznetsov, "Domain patterning by electron beam of mgo doped lithium niobate covered by resist," Applied Physics Letters, vol. 106, no. 23, p. 232902, 2015. [Online]. Available: https://doi.org/10.1063/1.4922372

[21] M. Bortz, S. Field, M. Fejer, D. Nam, R. Waarts, and D. Welch, "Noncritical quasi-phase-matched second harmonic generation in an annealed proton-exchanged $\mathrm{LiNbO} / \mathrm{sub} 3 /$ waveguide," IEEE $J$. Quantum Electron., vol. 30, no. 12, pp. 2953-2960, 1994. [Online]. Available: http://ieeexplore.iee.org/document/362710/

[22] M. Polyanskiy, "Refractiveindex.info," Available at: https: //refractiveindex.info, 2008-2021.

[23] K. El Hadi, V. Rastogi, M. R. Shenoy, K. Thyagarajan, M. De Micheli, and D. B. Ostrowsky, "Spectral measurement of the film-substrate index difference in proton-exchanged LiNbO_3 waveguides," Appl. Opt., vol. 37, no. 27, p. 6463, sep 1998. [Online]. Available: https://www.osapublishing.org/abstract.cfm?URI=ao-37-27-6463

[24] "Python software foundation. python language reference, version 2.7." Available at: http://www.python.org, 2010.

[25] L. Bolla, "Empy-electromagnetic python," Available at: https://github. com/lbolla/EMpy, 2017.

[26] Y. N. Korkishko and V. A. Fedorov, Ion Exchange in Single Crystals for Integrated Optics and Optoelectronics. Cambridge International Science Publishing, 1999.

[27] N. A. Sanford and W. C. Robinson, "Secondary-ion mass spectroscopy characterization of proton-exchanged LiNbO_3 waveguides," Opt. Lett., vol. 10, no. 4, p. 190, apr 1985. [Online]. Available: https://www.osapublishing.org/abstract.cfm?URI=ol-10-4-190

[28] D. Castaldini, P. Bassi, S. Tascu, P. Aschieri, M. P. D. Micheli, and P. Baldi, "Soft-proton-exchange tapers for low insertion-loss linbo3 devices," J. Lightwave Technol., vol. 25, no. 6, pp. 1588-1593, Jun 2007. [Online]. Available: http://jlt.osa.org/abstract.cfm?URI=jlt-25-6-1588

[29] T. Lunghi, F. Doutre, A. P. Rambu, M. Bellec, M. P. De Micheli, A. M. Apetrei, O. Alibart, N. Belabas, S. Tascu, and S. Tanzilli, "Broadband integrated beam splitter using spatial adiabatic passage," Opt. Express, vol. 26, no. 21, p. 27058, oct 2018. [Online]. Available: https://www.osapublishing.org/abstract.cfm?URI=oe-26-21-27058 